\title{
Effects of water immersion on quasi-static standing exploring center of pressure sway and trunk acceleration: a case series after incomplete spinal cord injury
}

\author{
Andresa R. Marinho-Buzelli $\mathbb{\Phi}^{1,2} \cdot$ Hossein Rouhani ${ }^{3} \cdot$ Beverley Catharine Craven $\mathbb{(}^{1,4} \cdot$ Kei Masani $\mathbb{C}^{1,5} \cdot$ \\ José Angelo Barela ${ }^{6} \cdot$ Milos R Popovic $^{1,7} \cdot$ Mary C. Verrier ${ }^{1,8}$
}

Received: 15 August 2018 / Revised: 24 November 2018 / Accepted: 2 January 2019

(c) International Spinal Cord Society 2019

\begin{abstract}
Study design This work is a case series.

Objectives We assessed the influence of the aquatic environment on quasi-static posture by measuring center of pressure (COP) sway and trunk acceleration parameters after incomplete spinal cord injury (iSCI) in water and on land.

Setting Tertiary Rehabilitation Hospital, Ontario, Canada.

Methods Six adult participants with iSCI ( 4 cervical/2 thoracic injuries, AIS D) were enrolled. Baseline balance was assessed by the Berg Balance Scale and Mini-Balance Evaluation System Test. Participants stood on a waterproof force plate for one minute per trial on land and in water; participants completed testing with their eyes open or closed in random order over 10 trials. Individuals' perceptions of their standing balance were obtained. COP and trunk acceleration parameters were analyzed in the time-domain.

Results COP sway and upper to lower trunk acceleration ratios in the AP direction increased in water, which was in contrast to standing on land in both visual conditions for 5/6 participants. Three participants (P1, P3 \& P4) with greater sensorimotor deficits had larger COP sway in water with the eyes closed. Two (P1 \& P4) of six participants reported more discomfort standing in water than standing on land.

Conclusions Increased COP sway seemed to reflect the balance and sensorimotor impairments of the participants, especially when standing with eyes closed in water. Although most participants $(4 / 6)$ perceived that they swayed more in water in contrast to on land, 5 out of 6 participants reported that water felt like a safer environment in which to stand.
\end{abstract}

Supplementary information The online version of this article (https:// doi.org/10.1038/s41394-019-0147-2) contains supplementary material, which is available to authorized users.

Andresa R. Marinho-Buzelli

andresa.marinho@mail.utoronto.ca

1 Toronto Rehabilitation Institute - University Health Network, Toronto, ON, Canada

2 Rehabilitation Sciences Institute - University of Toronto, Toronto, ON, Canada

3 Department of Mechanical Engineering, University of Alberta, Edmonton, AB, Canada

4 Department of Medicine, Rehabilitation Sciences Institute University of Toronto, Toronto, ON, Canada

\section{Introduction}

Aquatic therapy has been underexplored in spinal cord injury (SCI) rehabilitation, despite the purported benefits of water immersion for the SCI population [1,2]. Although evidence supporting the positive effects of aquatic therapy on mobility among individuals with incomplete SCI (iSCI)

5 Institute of Biomaterials and Biomedical Engineering, University of Toronto, Toronto, ON, Canada

6 Institute of Biosciences - Sao Paulo State University, Rio Claro, Sao Paulo, Brazil

7 Institute of Biomaterials and Biomedical Engineering, Rehabilitation Sciences Institute - University of Toronto, Toronto, ON, Canada

8 Department of Physical Therapy, Rehabilitation Sciences Institute, Institute of Medical Science, University of Toronto, Toronto, ON, Canada 
is scarce [3], aquatic therapy has emerged as a promising approach to augment balance recovery [4]. Stevens et al. (2015) reported improvements in balance function post underwater treadmill training in adults with iSCI [4]. The physical properties of water have the potential to benefit individuals with iSCI by providing a reduction of apparent body weight due to buoyant force, strengthening muscles due to water resistance, and augmenting balance control due to the stimuli associated with water turbulence when moving in the pool [5].

We investigated the performance of quasi-static standing posture during water immersion versus quasi-static standing on dry land. We expected that buoyancy would have a direct influence on balance control by offloading about $50 \%$ of the body weight with immersion around the umbilicus; and that balance control would be different from standing on land where participants bear their full body weight. We used the centre of pressure (COP) sway during quasi-static standing to describe and investigate postural balance among individuals with iSCI [6-8]. According to Tamburella et al., mean velocity or path length of COP, are most reliable and can reveal the balance impairment due to sensory and/or motor impairments that occur after iSCI [8]. We also examined the acceleration of the upper (xiphoid level) and lower trunk (lumbosacral joint level) during quiet standing to investigate whether immersion in water alters trunk movement during body sway [9]. On land, trunk acceleration has shown to be a sensitive, valid and reliable measure of mild postural balance impairments in individuals with Parkinson's Disease [10, 11].

Qualitative reporting of individuals' perceptions while standing in water and on land was used to enhance the interpretation of the quantitative parameters of postural sway. A mixed-methods approach has been reported to enhance the depth of the understanding of research questions and to assist the researcher to explore the meaning of the quantitative data [12].

This case series describes quantitative parameters and qualitative perceptions of quasi-static standing balance on dry land and during water immersion among adult participants with iSCI. We aim to inform aquatic rehabilitation practices by determining how water immersion influences the control of quasi-static balance after iSCI.

\section{Methods}

\section{Participants and location}

Six adults with iSCI ( 4 males $/ 2$ females, 4 cervical $/ 2$ thoracic injuries, AIS D, from 2 to 145 months post-injury) were recruited through poster advertisements at a tertiary SCI rehabilitation centre in Canada. Eligible participants with iSCI of traumatic or non-traumatic etiology, able to stand with eyes open and with eyes closed without assistance for at least $1 \mathrm{~min}$, were included. Participants were excluded if they had untreated mental disorder (e.g. anxiety or mood disorders creating water phobias), and respiratory, cardiac or skin diseases that contra-indicated immersion in warm water; or participants who required assistance to stand unsupported on land and in water, or had an allergy to chlorine. Six adults with iSCI consented to study participation during a ten-month period. Informed consent was obtained from each participant. This study has been performed in accordance with the Declaration of Helsinki and ethical approval was obtained from both the University Health Network and University of Toronto (REB 10-029).

\section{Clinical examination}

An evaluation of the patient's history and baseline clinical evaluations were complete one week prior to testing on land. The participants' neurological impairments were described using the International Standards for Neurological Classification of Spinal Cord injury (ISNCSCI) [13] by an expert physiotherapist trained in ISNCSCI assessment. The presence or absence of deep anal pressure and voluntary anal contraction was obtained through interview. The reported neurological level of injury and AIS classifications were verified using the online calculator developed by the Rick Hansen Institute based on the American Spinal Injury Association's (ASIA) ISNCSCI Revised 2011 [14].

The Berg Balance Scale (BBS) [15] and the Mini Balance Evaluation Systems Test (Mini-BESTest) [16] were used to characterize the clinical balance function of participants within quasi-static and dynamic domains of posture, prior to the tests in water and on land, assisting with the interpretation of the results from the COP sway and trunk acceleration. The BBS has been previously validated in the SCI population [17]. However, it has been reported that the BBS has a ceiling effect in patients with AIS D impairment [18]. The Mini-BESTest is a 14-item task-oriented scale focused on dynamic balance and developed to analyze postural control in four major domains: anticipatory, reactive postural control, sensory orientation and dynamic gait [16]. The Mini-BESTest has been validated in individuals with SCI and does not have ceiling effects in chronic SCI [19].

Anthropometric and neurological data for participants are displayed in Table 1 and the clinical balance scores are displayed in Table 2.

\section{Instrumentation}

Tests in water and on dry land occurred in the therapy pool area, in order to maintain the same experimental conditions 
Table 1 Participants' characteristics

\begin{tabular}{|c|c|c|c|c|c|c|}
\hline \multirow[t]{2}{*}{ Participants characteristics } & \multicolumn{6}{|l|}{ Participants } \\
\hline & 1 & 2 & 3 & 4 & 5 & 6 \\
\hline Age (years) & 61 & 69 & 42 & 60 & 53 & 56 \\
\hline Gender $(\mathrm{M} / \mathrm{F})$ & Male & Male & Male & Female & Female & Male \\
\hline Height $(\mathrm{cm})$ & 177.8 & 174.0 & 180.3 & 162.6 & 157.5 & 180.3 \\
\hline Body weight on land $(N)$ & 784.6 & 568.7 & 720.9 & 865.5 & 484.8 & 676.3 \\
\hline Body weight in water $(N)$ & 480.1 & 324.0 & 417.3 & 323.7 & 182.8 & 355.9 \\
\hline$\%$ of body weight offloading & $38.8 \%$ & $43.0 \%$ & $42.1 \%$ & $62.6 \%$ & $62.3 \%$ & $47.4 \%$ \\
\hline Mechanism of etiology & Non-traumatic & Traumatic & Non-traumatic & Non-traumatic & Traumatic & Traumatic \\
\hline Time since injury/surgery (months) & 2 & 3 & 64 & 145 & 76 & 3 \\
\hline Neurological level & $\mathrm{C} 1$ & C6 & $\mathrm{T} 10$ & $\mathrm{~T} 10$ & $\mathrm{C} 4$ & $\mathrm{C} 4$ \\
\hline AIS Scale & $\mathrm{D}$ & $\mathrm{D}$ & $\mathrm{D}$ & $\mathrm{D}$ & $\mathrm{D}$ & $\mathrm{D}$ \\
\hline Upper limb motor score $($ Right $/$ Left $=25 / 25)$ & $25 / 19$ & $21 / 21$ & $25 / 25$ & $25 / 25$ & $22 / 25$ & $20 / 20$ \\
\hline Lower limb motor score $($ Right/Left $=25 / 25)$ & $25 / 22$ & $25 / 22$ & $25 / 24$ & $25 / 17$ & $24 / 24$ & $23 / 23$ \\
\hline Light touch $($ Right $/$ Left $=56 / 56)$ & $22 / 21$ & $54 / 49$ & $51 / 47$ & $50 / 46$ & $46 / 47$ & $32 / 31$ \\
\hline Pin prick $($ Right/Left $=56 / 56)$ & $9 / 21$ & $56 / 56$ & $48 / 39$ & $46 / 45$ & $36 / 37$ & $32 / 31$ \\
\hline Mobility function & Walking & Wheeling & Walking & Wheeling & Walking & Walking \\
\hline Assistive device for mobility & Cane & Power Wheelchair & Cane & Power Wheelchair & None & Rollator \\
\hline
\end{tabular}

Table 2 Classification of clinical balance of participants

\begin{tabular}{lrrrrrr}
\hline Balance scales & \multicolumn{7}{l}{ Participants } \\
\cline { 2 - 7 } & 1 & 2 & 3 & 4 & 5 & 6 \\
\hline Mini-BESTest & 2 & 2 & 4 & 2 & 5 & 4 \\
Anticipatory (0-6) & 1 & 1 & 3 & 0 & 5 & 6 \\
Reactive Postural Control (0-6) & 5 & 4 & 5 & 0 & 6 & 6 \\
Sensory orientation (0-6) & 8 & 1 & 7 & 1 & 8 & 10 \\
Dynamic Gait (0-10) & 16 & 8 & 19 & 3 & 24 & 26 \\
Mini-BESTest (Total: 0-28) & 51 & 42 & 45 & 40 & 55 & 56 \\
Berg Balance Scale (Total: $0-56)$ & & & & & & \\
\hline
\end{tabular}

for both tests (Fig. 1). The exact same instrumentation was used in the water and on land [9]. A waterproof force plate (AMTI, ORP-WP-1000, Watertown, USA) was used to collect COP parameters during quiet standing at a sampling frequency of $1000 \mathrm{~Hz}$ (Fig. 1). Two wireless body-worn inertial sensors (Physilog, BioAGM, Lausanne, Switzerland) sealed in waterproof bags were attached to the lower trunk region (L5/S1) and to the upper trunk region (head of sternum) to collect data synchronously at a sampling frequency of $500 \mathrm{~Hz}$.

\section{Experimental procedure}

Tests on land and in water were performed on two consecutive days, except for P3 who performed the tests on day 1 and day 3 due to logistical constraints. In water, participants were immersed at the umbilicus level, in the deep end of the therapy pool, which favored the analysis of upper and lower trunk dynamics, where the lower trunk was immersed up to the umbilicus (at the level of $3^{\text {rd }}$ and $4^{\text {th }}$ lumbar vertebrae) and the upper trunk was above the water surface. Participants were instructed to stand "as quiet as possible" on the force plate, in their chosen comfortable position, with their arms crossed in front of their chest, standing with their same foot placement across all trials (Fig. 1), on land and in water. The outer perimeter of both feet was drawn on a force plate in order for participants to stand using their same base of support (Fig. 1). A fixed target was positioned at eye level approximately 2-m distance away from the participant. Visual condition was randomly assigned to 10 trials, five with the eyes open and five with the eyes closed. Each trial lasted $60 \mathrm{sec}$ and participants were allowed to rest on a chair between trials, as needed, to avoid the influence of fatigue.

After completing the tests on land and in water, participants were asked about their perceptions of standing in water and on land (Appendix). The individuals' perceptions were annotated by the examiner.

\section{Data analysis}

The percentage of body weight (BW) offloading (\%offloading) when participants were immersed in water was calculated using the following: \%offloading $=100 \times$ $\left(\mathrm{BW}_{\text {land }}-\mathrm{BW}_{\text {water }}\right) / \mathrm{BW}_{\text {land, }}$ where: $\mathrm{BW}_{\text {land }}$ was the body 


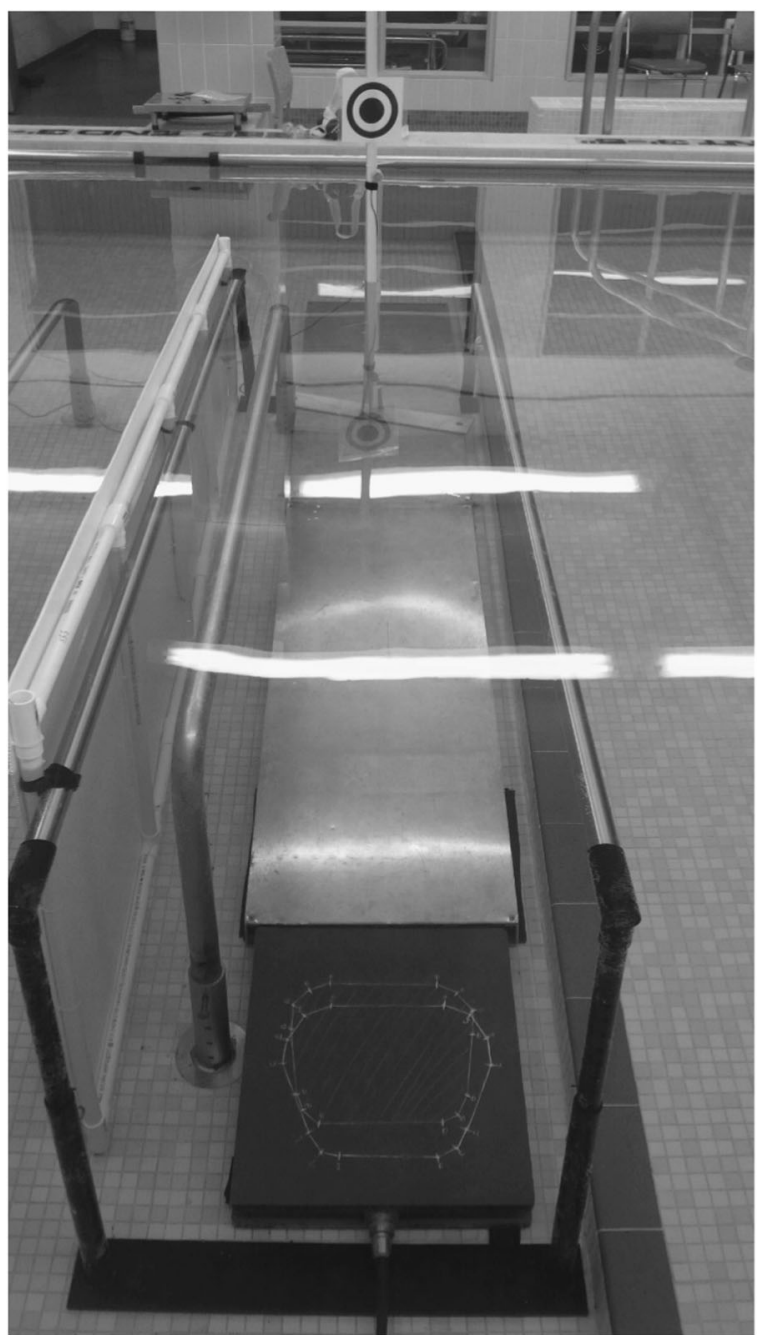

Fig. 1 Experimental set in a therapy pool measuring $9.80 \mathrm{~m}$ long, $4.90 \mathrm{~m}$ wide and $1.10 \mathrm{~m}$ deep (deepest end). The experiment occurred in the deepest end of the pool. Participants stood on a waterproof force plate $(0.50 \times 0.46 \times 0.08 \mathrm{~m})$, within demarked feet contour, in all trials in water and on land. The figure displays the feet contour for two participants. When standing with eyes open, a target was placed at 2-m distance away from participants

weight on dry land and $\mathrm{BW}_{\text {water }}$ was the apparent body weight in water measured by the force plate. $\mathrm{BW}_{\text {land }}$ and $\mathrm{BW}_{\text {water }}$ was determined through the calculation of the mean vertical force on the force plate for the period of $55 \mathrm{sec}$ of quiet standing on land and in water, respectively.

We analyzed $55 \mathrm{sec}$ of quiet standing from $60 \mathrm{~s}$ of collected data. We eliminated the initial and final $2.5 \mathrm{sec}$ of the trials to avoid initial and final transient and anticipation effects. The time series of COP and accelerometer readout (acceleration) were filtered using a 4th order and zero-lag Butterworth digital filter with a low-pass, cut-off frequency of $5 \mathrm{~Hz}$. The acceleration time series was also high pass filtered at $0.15 \mathrm{~Hz}$ [9].
The COP parameters (e.g. root mean square - RMS, mean velocity - MVELO, and sway area) were used to quantify the COP sway in this study. The acceleration parameters (RMS and upper to lower trunk RMS ratio) were calculated as reported in Marinho-Buzelli et al. [9]. The ratio of upper trunk to lower trunk acceleration was used to determine whether there was a change in the pattern of acceleration between upper trunk and lower trunk during quiet standing [9].

Descriptive statistics (median and interquartile range) were applied to summarize the dependent variables of all trials in each condition. A thematic analysis approach was used to analyze data from open-ended questions [20].

We certify that all applicable institutional and governmental regulations concerning the ethical use of human volunteers were followed during the course of this research.

\section{Results}

\section{Clinical balance}

The BBS scores ranged from 40 to 56 among the participants (Table 2). Only P4 scored 40 points, which suggests increased risk of falling. P4 did not demonstrate reactive postural control or sensory orientation and presented low dynamic gait scores on the Mini-BESTest. P2 also had low dynamic gait and reactive postural control scores. P2 and P4 with low dynamic gait scores were the ones who used a power wheelchair for mobility. P5 and P6 approached a ceiling effect on balance performance for the BBS, scoring 55 and 56, respectively, and reported to be walkers, one with no assistive device (P5) and one with a rollator walker (P6).

\section{COP parameters}

Overall, each participant presented larger medians of COP parameters in water contrasted to land both with eyes open and eyes closed (Table 3). P3 had the most accentuated COP displacement with eyes closed in water in comparison to land, and P4 presented the largest sway area in water with eyes closed (Table 3). P1, with a moderate light touch and pinprick sensory deficit (Table 1), had the most accentuated increase of COP displacement (Fig. 2a) and velocity (Fig. 2b) in his first trial in water contrasted to land, when his eyes were closed. $\mathrm{P} 3$ and $\mathrm{P} 4$, who presented with relatively low balance scores (Table 2) and P1 with moderate sensory deficit (Table 1) had an accentuated increase in RMS-COP and MVELO in AP direction in the water in contrast with participants with high balance functioning (P5 and P6) (Fig. 2a and Fig. 2b). 


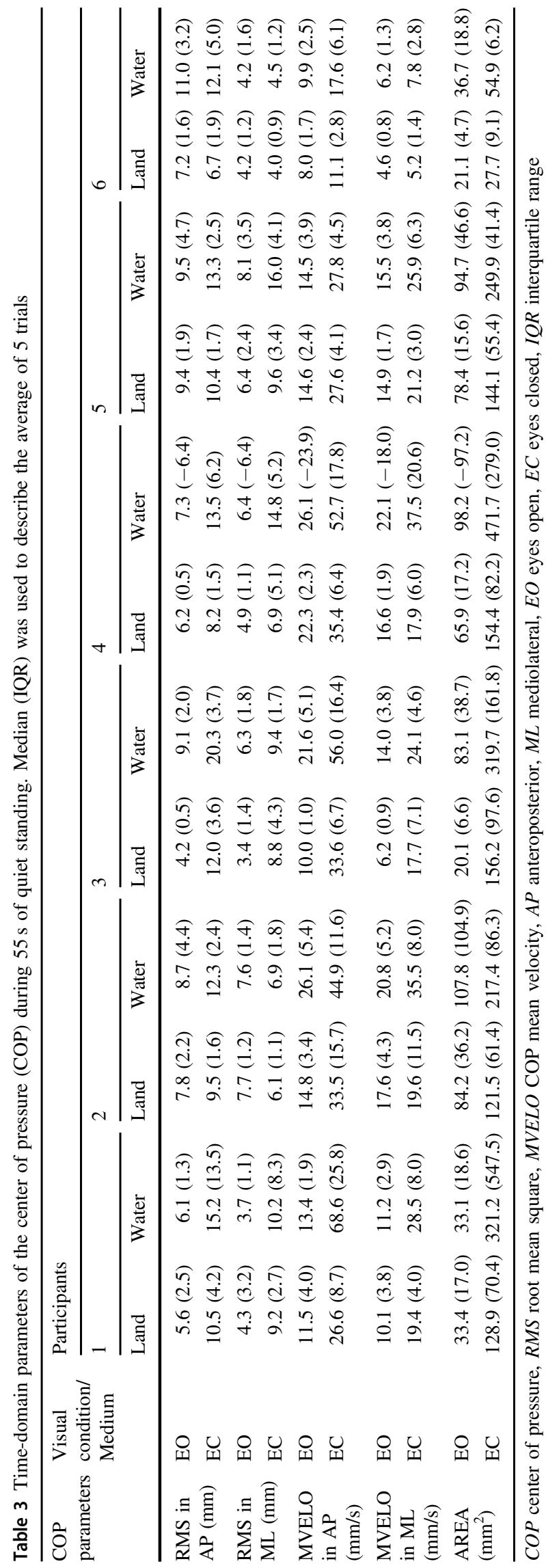

\section{Trunk acceleration parameters}

The RMS acceleration of the upper and lower trunk varied among participants, as shown in Table 4. Four participants (P2, P4, P5 and P6) presented smaller medians of RMS acceleration in AP direction in the upper and lower trunks in water in contrast to dry land, in most visual conditions. Only P1, with a moderate sensory deficit, presented increased RMS acceleration in water contrasted to land in the lower trunk in AP and ML directions, when eyes were closed (Table 4).

Although the medians of RMS acceleration in the upper and lower trunk of four participants (P2, P4, P5 and P6) seemed to be smaller in water than on land in the AP direction in most visual conditions, three of these participants presented larger AP upper to lower trunk acceleration ratio in most trials with eyes closed, in water in contrast to land (P2, P4 and P5) (Fig. 3).

\section{Participants perception while standing in water and on land}

The reported perceptions of the participants indicate that their neurological status and clinical balance function may have influenced their perceptions after standing in water and on dry land. For example, P1 with moderate to severe light touch and pin prick deficits (Table 1) reported, "when my eyes were closed, I had difficulty maintaining a still position in water". His postural strategy was to "balance more on the balls of his feet" to maintain an upright position in water with eyes closed. A slight movement in the water led to loss of balance as suggested by his report, "In water, the slightest movement knocked me back. Just the smallest vibration could cause balance loss during eyes closed. I had no issues with eyes open". However, this participant mentioned "falling in water I wouldn't hurt myself $[\ldots]$ the buoyancy would prevent injury".

In contrast with P1, P2 who presented with a slight sensory deficit (light touch and pin prick) (Table 1) and relatively low balance scores (Table 2), reported "my balance was better in the water especially when I closed my eyes. I felt I could stay straighter in the water without losing my balance. On land, I was more inclined forward". He further reported "I felt a little less stable in the water because the movement of water in the beginning. I felt more comfortable and confident as we went on. I felt better in the water than on land".

As for P3, who had chronic pain, he reported that "the water was more relaxing, more calming. I have 7 and 8 pain level on land. In the water it is 4 and 5 . Specially because the temperature in the water. My pain levels are much less". P3 had no concerns regarding falling in water when his eyes were closed as he reported, "when I have to close my eyes, I 
Fig. 2 a Participants (P1 to P6) root mean square of the center of pressure (RMS-COP) in the anteroposterior (AP) direction across five trials of quiet standing with eyes closed (EC) condition, on land (black) and in water (gray). b Participants (P1 to P6) mean velocity of the center of pressure (MVELO) in AP direction across five trials of quiet standing with eyes closed (EC) condition, on land (black) and in water (gray)
A

RMS-COP in AP direction with EC (mm)
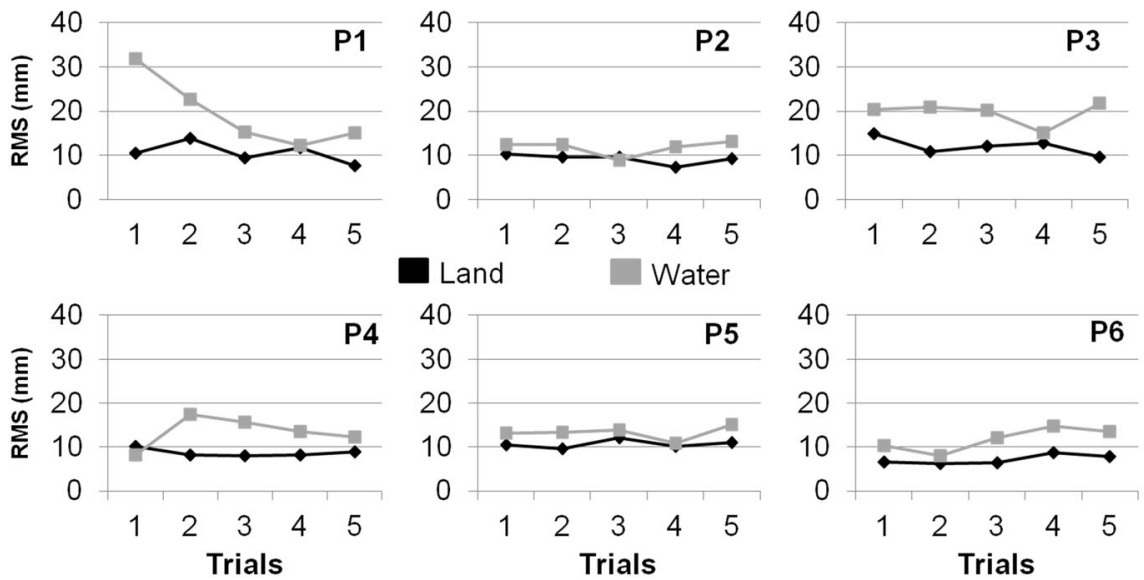

B

MVELO in AP direction with EC $(\mathrm{mm} / \mathrm{s})$
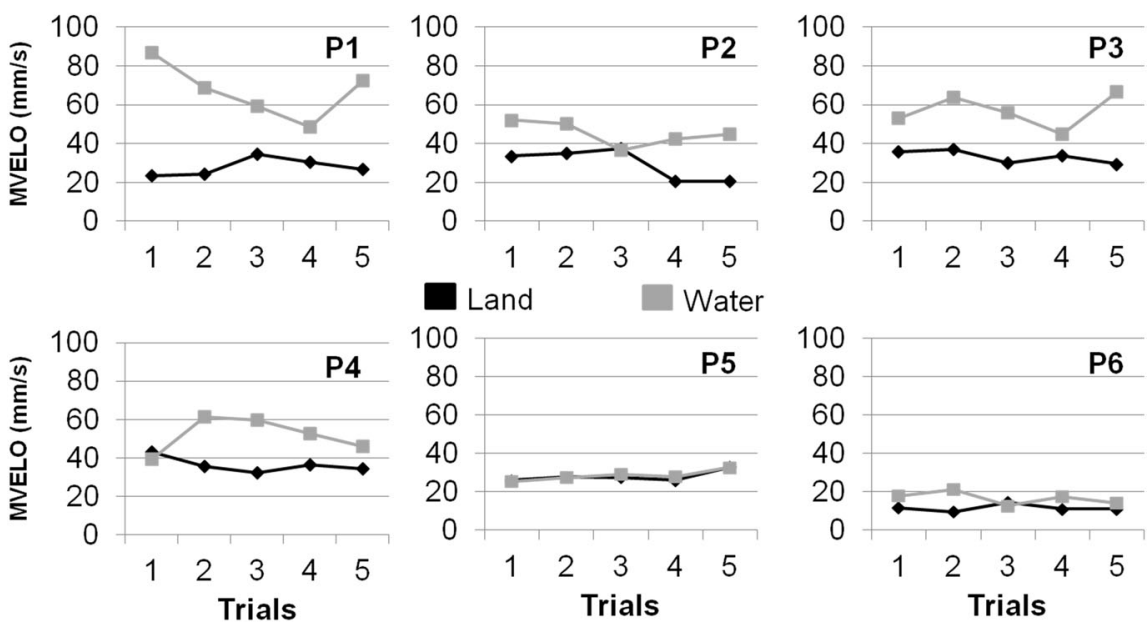

was scared to fall on land. I have no concern in water while standing". He also reported that standing in water was less tiring, "effortless in water. Less tiring. Less breaks. For sure less tiring." P4, who presented with low balance function and hypertonia in the plantar flexion, found the water a very difficult environment to maintain balance. She said "I was not sure of my footing in water. Heel was up a bit". Thus, for her, standing on land appeared to be much easier, "on land I could balance better... not holding and crossing arms it was easier on land."

\section{Discussion}

This case series reports the influence of water immersion on the control of COP sway and trunk acceleration for iSCI among six individuals with diverse etiology, impairments and functional abilities. Overall, we found that the medians of COP displacement, velocity, and area for individuals with iSCI were larger in water in contrast with dry land, in both visual conditions (eyes open and closed). Although trunk acceleration in the AP direction decreased in water in four out of six participants, the ratio of upper trunk to lower trunk acceleration of three participants increased in water in contrast to land indicating a potential change and suggesting they may have used an adaptive trunk strategy to maintain their balance in water. The observed results concur with previous studies among able-bodied participants [9, 21, 22].

\section{The influence of visual input on the COP sway in water}

The observation of the influence of visual input in the two environments, by contrasting the medians in all environmental and visual conditions, showed that P1, P3 and P4 also had larger COP AP displacement and velocity with eyes closed in water compared to the eyes closed condition on land. It is unclear if there is a dominant factor causing the potential increased instability in water with eyes closed. The clinical assessments demonstrated that P1 had a moderate 


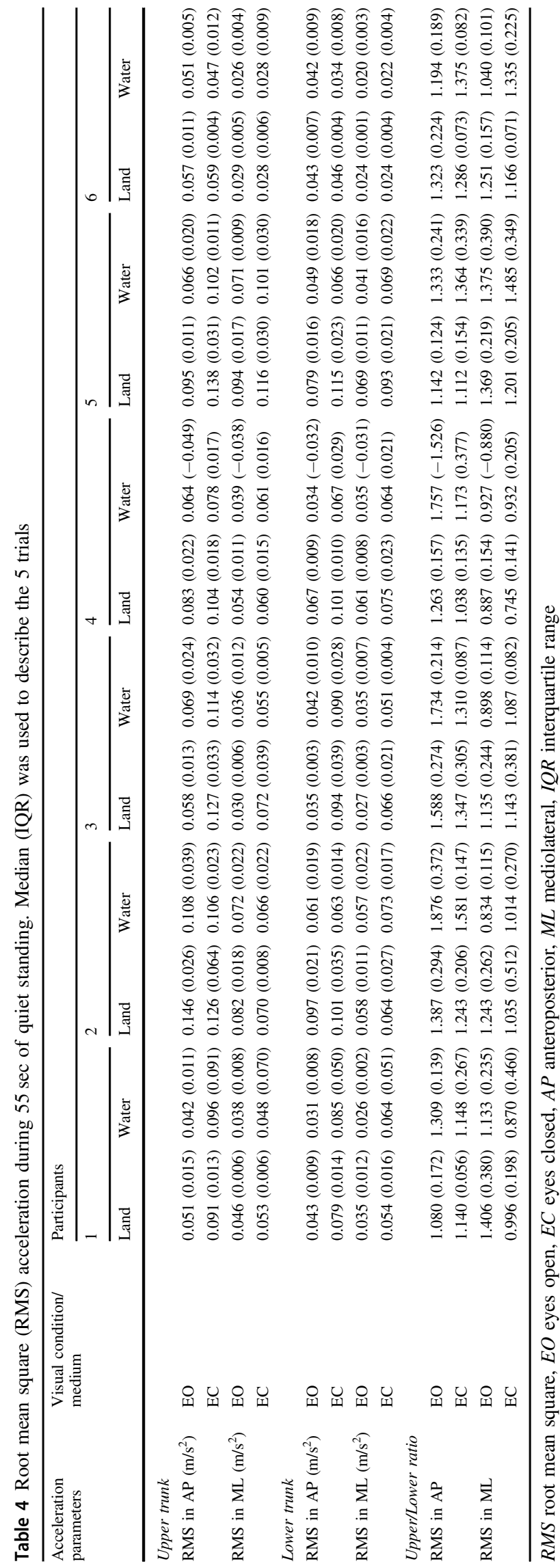

sensory deficit (pinprick and light touch) aligned with poor reactive postural control on Mini-BESTest; P3 had a selfreported moderate chronic pain, also aligned with low reactive balance control on Mini-BESTest; and $\mathrm{P} 4$ reported having increased tone and spasms in the lower limbs, with poor overall balance control, on Mini-BESTest and BBS scales. We are uncertain if any condition and which of these conditions led to increased COP sway in water with eyes closed as other factors, e.g. trunk muscle strength, could potentially be affecting postural control across different levels of neurological impairment.

\section{Influence of neurological and functional status on perceptions of body sway in water}

The collective findings for these six individuals with diverse neurological impairments, mobility and balance capacity support our understanding of how postural responses to immersion in water seem to be related to the neurological and functional status of each participant. For example, P1, who presented with a moderate sensory deficit (light touch and pinprick) 2 months after injury and had low anticipatory and reactive balance on the Mini-BESTest reported, "in water, the biggest challenge was keeping a still position. On dry land, I felt there were no issues, didn't lose balance." Although it is unknown if his sensory deficit played a major role in his postural instability, his report of losing balance in the water was consistent with the observed accentuation of COP sway in water with eyes closed, when he was devoid of both visual and sensory input, especially in his first trial.

In contrast, $\mathrm{P} 2$, who presented with low balance function on the clinical measures but preserved sensory function, perceived an increased sway in water. However, P2 seemed to have adapted to the water environment differently, and reported, "I felt I could stay more straight in water without losing my balance. On land, I was more inclined forward".

\section{Is the aquatic environment a stimulus for training postural control?}

The increased COP sway (displacement and velocity) during pool immersion may reflect decreased postural steadiness and/or greater stimuli for postural control, as a result of the postural disturbances in the aquatic environment. Aquatic therapy programs focused on balance and mobility may have contributed to reported gains in balance function in other neurological populations [3, 23]. In other populations with disabilities, aquatic exercise programs have led to improvements in parameters of COP sway on dry land. For example, total sway area and lateral sway were decreased after aquatic intervention in woman with arthritis [24]. Berger et al. have reported increased postural stability after one session of aquatic therapy on a group of people with 
Fig. 3 Upper to Lower trunk root mean square acceleration (RMS-ACC) ratio in anteroposterior (AP) direction of participants (P1 to P6) five trials of quiet standing with eyes closed (EC) condition, on land (black) and in water (gray)
Upper/Lower trunk RMS-ACC in AP direction with EC
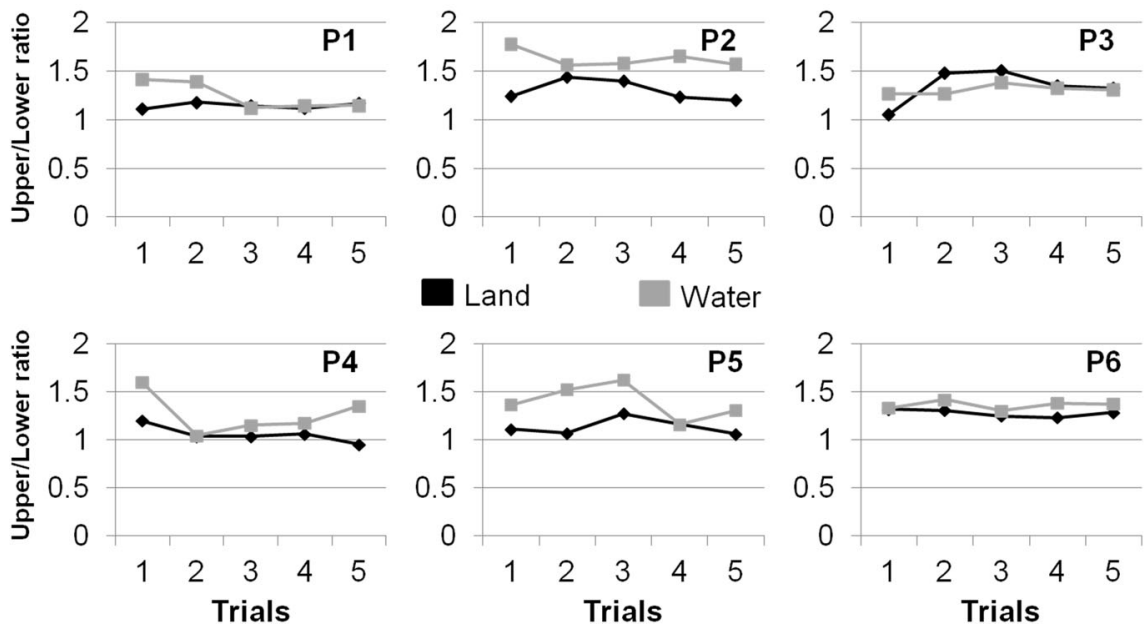

lower limb injuries [25]. Further studies are warranted to determine if the response evoked in water by the mechanical and sensory perturbations, i.e. by causing a larger COP sway and changing the upper to lower trunk acceleration pattern, have the potential to augment balance among individuals with iSCI and impairments in postural control. The differences in performance of these heterogeneous individuals with iSCI along the differences within trials suggest that more than five trials for each visual condition may be required to distinguish the differences among water and land environments in future cross-sectional studies.

\section{Other contributors for postural control}

The present study has some limitations and is not able to inform us whether other major determinants of postural control, such as trunk strength and control, affected the participants balance control [26]. Future clinical trials investigating the effects of aquatic therapy paradigms to address stability for individuals with SCI with predetermined levels of balance control under different aquatic conditions and different postural positions for the upper extremities, would advance our understanding of postural control in water. Intervention studies investigating whether aquatic therapy is superior and/or an adjunct to land therapy in the recovery of balance of individuals with iSCI are warranted.

\section{Concluding remarks}

This case series revealed that not all individuals felt comfortable immersed in the water. Particularly, P1 with sensory deficits and P4 with spasticity, both having very low reactive balance control, felt they were more comfortable while standing on land, as evidenced by their qualitative reporting. Although two participants preferred standing on land than in water, five out of six participants felt more secure standing in water than on land, since they did not have the fear of falling. Clearly, assessing self-efficacy in behavioral paradigms of balance control needs to be incorporated into future studies.

Our findings are important to understand the mechanisms of immersion while standing in water, i.e. quantifying the dynamics of upper and lower trunk movement, and the whole body response to immersion as measured by the COP parameters. Our study showed that upper and lower trunk acceleration ratio of three participants changed, indicating a new postural strategy to maintain balance in water, with about $50 \%$ of the body weight offloaded and the lower body (up to the umbilicus) feeling the effects of the hydrostatic pressure and thermic effects of water. The increased displacement and velocity of the COP seems to be a response for postural control, which could benefit balance gains on land. Therefore, understanding how posture is changed by the immersion in water is informative to future clinical studies, especially those with focus on regaining balance control. Collectively, participants felt they swayed more in water than on land and found water a safe environment for standing. Having patients with SCI stand in water experiencing postural perturbations, even beyond their limits of stability, may be an important rehabilitative approach to challenge balance control and facilitate balance recovery.

Acknowledgements We thank the expert physiotherapist Chris Allapat for his collaboration in assessing the neurological level and clinical balance of the participants of the present study. The authors thank Dr. Kamiar Aminian for providing this research with the inertial sensor system. We also acknowledge the research and technical support of Adolazim Rashidi and Carlos Buzelli Neto during the experiments, and the assistance of the summer students from the SCI Mobility Laboratory at Toronto Rehabilitation Institute - University Health Network. 
Funding ARM-B acknowledges the support of Canadian Institutes of Health and Research (CIHR) through the Vanier Canada Graduate Scholarship on the development of this study [Grant \# 95662]. HR acknowledges the support of the Swiss National Science Foundation Grants [PBELP3-137539 and P300P2-147865] and Spinal Cord Injury Ontario Postdoctoral Fellowship. This research was also supported by the Natural Sciences and Engineering Research Council grant [\#249669], Ontario Neurotrauma Foundation, Réseau Provincial de Recherche en Adaptation-Réadaptation (REPAR) and Toronto Rehabilitation Institute - University Health Network.

\section{Compliance with ethical standards}

Conflict of interest The authors declare that they have no conflict of interest.

Publisher's note: Springer Nature remains neutral with regard to jurisdictional claims in published maps and institutional affiliations.

\section{References}

1. Recio AC, Stiens SA, Kubrova E. Aquatic-Based therapy in spinal cord injury rehabilitation: effective yet underutilized. Curr Phys Med Rehabil Rep. 2017;5:1-5.

2. Li C, Khoo S, Adnan A. Effects of aquatic exercise on physical function and fitness among people with spinal cord injury. Med (Baltim). 2017;96:1-6.

3. Marinho-Buzelli AR, Bonnyman AM, Verrier MC. The effects of aquatic therapy on mobility of individuals with neurological diseases: a systematic review. Clin Rehabil. 2015;29:741-51.

4. Stevens SL, Caputo JL, Fuller DK, Morgan DW. Effects of underwater treadmill training on legstrength, balance, and walking performance in adults with incomplete spinal cord injury. J Spinal Cord Med. 2015;38:91-101.

5. Becker B, Cole A. Comprehensive aquatic therapy. 3rd ed. Pullman, Washington: State University Publishing; 2011.

6. Arora T, Musselman K, Lanovaz J, Oates A. Effect of haptic input on standing balance among individuals with incomplete spinal cord injury. Neurosci Lett. 2017;642:91-6.

7. Lemay JF, Gagnon D, Duclos C, Grangeon M, Gauthier C, Nadeau S. Influence of visual inputs on quasi-static standing postural steadiness in individuals with spinal cord injury. Gait Posture. 2013;38:357-60.

8. Tamburella F, Scivoletto G, Iosa M, Molinari M. Reliability, validity, and effectiveness of center of pressure parameters in assessing stabilometric platform in subjects with incomplete spinal cord injury: a serial cross-sectional study. J Neuroeng Rehabil. 2014;11:86

9. Marinho-Buzelli AR, Rouhani H, Masani K, Verrier MC, Popovic MR. The influence of the aquatic environment on the control of postural sway. Gait Posture. 2017;51:70-6.

10. Horak F, King L, Mancini M. Role of body-worn movement monitor technology for balance and gait rehabilitation. Phys Ther. 2015;95:461-70.
11. Mancini M, Salarian A, Carlson-Kuhta P, Zampieri C, King L, Chiari L, et al. ISway: a sensitive, valid and reliable measure of postural control. J Neuroeng Rehabil. 2012;9:59.

12. Rauscher L, Greenfield BH. Advancements in contemporary physical therapy research: use of mixed methods designs. Phys Ther. 2009;89:91-100.

13. Kirshblum SC, Waring W, Biering-Sorensen F, Burns SP, Johansen M, Schmidt-Read M, et al. International standards for neurological classification of spinal cord injury. J Spinal Cord Med. 2011;34:535-46.

14. Walden K, Bélanger LM, Biering-Sørensen F, Burns SP, Echeverria $\mathrm{E}$, Kirshblum $\mathrm{S}$, et al. Development and validation of a computerized algorithm for International Standards for Neurological Classification of Spinal Cord Injury (ISNCSCI). Spinal Cord. 2016;54:197-203.

15. Berg K. Measuring balance in the elderly: development and validation of an instrument. Can J Public Health. 1992;83(suppl 2):S7-11.

16. Franchignoni F, Horak F, Godi M, Nardone A, Giordano A. Using psychometric techniques to improve the balance evaluation systems test: The Mini-BESTest. J Rehabil Med. 2010;42:323-31.

17. Spinal Cord Injury Research Evidence. https://scireproject.com/ outcome-measures/outcome-measure-tool/berg-balance-scale-bbs/ . [Accessed $4^{\text {th }}$ February 2016].

18. Lemay J-F, Nadeau S. Standing balance assessment in ASIA D paraplegic and tetraplegic participants: concurrent validity of the Berg Balance Scale. Spinal Cord. 2010;48:245-50.

19. Jorgensen V, Opheim A, Halvarsson A, Franzen E, Roaldsen K. Comparison of the Berg Balance Scale and MIni-BESTest for assessing balance in ambulatory people with Spinal Cord Injury: validation study. Phys Ther. 2017;97:677-87.

20. Braun V, Clarke V. Using thematic analysis in psychology. Qual Res Psychol. 2006;3:77-101.

21. Louder T, Bressel E, Baldwin M, Dolny DG, Gordin R, Miller A. Effect of aquatic immersion on static balance. Int $\mathrm{J}$ Aquat Res Educ. 2014;8:53-65.

22. Schaefer SY, Louder TJ, Foster S, Bressel E. Effect of water immersion on dual-task performance: implications for aquatic therapy. Physiother Res Int. 2016;21:147-54.

23. Tripp F, Krakow K. Effects of an aquatic therapy approach (Halliwick-Therapy) on functional mobility in subacute stroke patients: a randomized controlled trial. Clin Rehabil. 2014;28:432-9.

24. Suomi R, Koceja DM. Postural sway characteristics of women with lower extremity arthritis before and after an aquatic exercise intervention. Arch Phys Med Rehabil. 2000;81:780-5.

25. Berger L, Martinie P, Livain T, Bergeau J, Rougier P. Effets immédiats de séances de rééducation des membres inférieurs par balneotherapie sur le contrôle de l'équilibre. Ann Readapt Med Phys. 2006;49:37-43.

26. Quinzanos-Fresnedo J, Fratini-Escobar PC, Almaguer-Benavides KM, Aguirre-Guemez AV, Barrera-Ortiz A, Perez-Zavala M, et al. Prognostic validity of a clinical trunk control test for independence and walking in individuals with spinal cord injury. J Spinal Cord Med. 2018. https://doi.org/10.1080/10790268.2018. 1518124. 